\title{
Saúde Mental e os Planos de Saúde no Brasil
}

\author{
Mental Health and Health Insurance Plans in Brazil
}

Paulo Fagundes da Silva ${ }^{1}$ Nilson do Rosário Costa ${ }^{1}$
${ }^{1}$ Departamento de Ciências Sociais, Escola Nacional de Saúde Pública, Fiocruz. Rua Leopoldo Bulhões 1480/ 912, Manguinhos. 21041-21 Rio de Janeiro RJ. paulofagundes@ensp.fiocruz.br
Abstract The article examines the regulatory regime of mental healthcare implemented by the National Health Agency (ANS). It describes the conditions observed between the provision of mental health services in the private healthcare sector in relation to the international experience and the Unified Health System (SUS). The article shows that the mental healthcare provision by the sector has the failings associated with the health insurance market. The health insurance companies have recourse to copayment mechanisms, set limits for outpatient use, emphasize short duration hospitalizations for the treatment of severe cases and residually offer support services to the patient after discharge. Since the decade of 2000 the expansion of the number of psychiatric hospitalizations that resulted in high rates of admission compared to the public sector has been observed in Brazil.

Key words Mental health, Health insurance, Prepaid health plans, Brazil, Psychiatric reform
Resumo $O$ artigo analisa o regime de regulação da assistência à saúde mental implantado pela Agência Nacional de Saúde Suplementar. Descreve as condições observadas entre a provisão de serviços de saúde mental no setor de planos privados de assistência à saúde em relação à experiência internacional e ao Sistema Único de Saúde (SUS). $O$ artigo demonstra que a assistência à saúde mental do setor apresenta as falhas associadas ao mercado de seguro saúde. As empresas de planos de saúde adotam mecanismos de copagamento, estabelecem limites de utilização para consultas ambulatoriais, enfatizam o tratamento dos casos graves por internação hospitalar de curta duração $e$ oferecem residualmente serviços de suporte ao paciente após a alta. Existem evidências de um descompasso entre o processo de desinstitucionalização em curso no SUS e a regulação implantada pela ANS. Em fins da década de 2000, foi observada a expansão da quantidade das internações psiquiátricas que resultaram em taxas elevadas de admissão se comparadas ao setor público no Brasil. Palavras Chaves Saúde mental, Seguros de saúde, Planos de Saúde Pré-pagos, Reforma Psiquiátrica 


\section{Introdução}

O objetivo deste artigo é descrever o regime de regulação da assistência à saúde mental pela Agência Nacional de Saúde Suplementar (ANS). O mercado de planos privados de assistência à saúde no Brasil está submetido a condições regulatórias específicas desde a implantação da Lei 9.656 em 1998 e a criação da ANS em 2000. O regime de regulação nasceu no mesmo contexto institucional da implantação da Lei 10.216, promulgada em 2001, pelo Sistema Único de Saúde (SUS). Quais as iniciativas desenvolvidas pela intervenção da ANS acompanham o modelo normativo presente na Lei 10.216/2001? A política de saúde mental no SUS tem buscado a mudança do modelo assistencial, privilegiando o direito individual do paciente e a interação com a família e a comunidade em lugar do isolamento. A reforma psiquiátrica tem sido bem sucedida na implantação de uma agenda estratégica de substituição progressiva dos hospitais psiquiátricos, pois busca a indução das internações necessárias em hospitais gerais e enfatiza o tratamento ambulatorial em novos serviços territoriais. A reforma psiquiátrica brasileira filia-se à agenda de transformação das instituições psiquiátricas clássicas no bojo do movimento de redemocratização e de ampliação dos direitos sociais no âmbito do setor público.

As motivações para a instituição de um regime de regulação para os planos de saúde no Brasil foram de outra natureza. Este mercado setorial desenvolveu-se em um contexto institucional de baixa regulação, até fins da década de 1990, pela ausência de barreiras de entrada e saída das empresas. Estas, também foram muito favorecidas por incentivos fiscais para à aquisição de seguro saúde por indivíduos e empresas.

Costa $^{1}$ chama atenção para as extraordinárias condições institucionais que possibilitaram a ampliação da clientela e a abertura de empresas de diferentes escalas, intermediadoras do acesso à atenção à saúde em todas as cidades de médio e grande porte. Por essa razão, o segmento de planos de assistência à saúde compreende até os dias atuais de um universo heterogêneo de modalidades organizacionais: empresas de medicina de grupo, empresas de autogestão (públicas e privadas), seguradoras e cooperativas médicas e odontológicas.

A instituição do novo marco pela Lei 9.656/ 1998 modificou significativamente esse cenário, pela definição de novas regras de proteção aos consumidores e de entrada, permanência e saída das empresas no mercado setorial ${ }^{2,3}$. No contexto anterior à atual regulação, os estudos sobre o setor identificavam problemas de exclusões e de variações no escopo e na natureza da cobertura em quase todos os planos privados, permitindo que opções desfavoráveis fossem oferecidas ao consumidor, acarretando uma lógica prevalente de seleção de risco.

Esta estratégia afetou os beneficiários de planos individuais que contraíssem enfermidades de alto custo ou de tratamento de longa duração, como o caso dos transtornos mentais, que não eram cobertos pela maioria dos contratos, e ficaram sob a gestão e a assistência do SUS. Esse fato fortaleceu a preocupação sobre as relações público e privado no âmbito da saúde, abrindo espaço para a regulação na perspectiva inicial do direito do consumidor e do respeito a contratos e, posteriormente, na construção de um regime regulatório que interfere fortemente sobre a atividade empresarial do setor de seguro saúde.

A intervenção normativa da ANS é, assim, orientada para garantir o desenvolvimento de novos contratos que contemplem a equidade nas condições de cobertura, a extinção dos limites de internação, a proibição de aumento por faixa etária após 60 anos com 10 anos de contribuição e a qualidade mínima nos padrões de atenção ${ }^{4}$.

Pretende-se demonstrar e discutir no presente artigo o padrão de resposta das empresas de planos de saúde ao regime de regulação e as principais diferenças e similitudes observadas entre a provisão de serviços de saúde mental do setor de saúde suplementar e o padrão normativo da política pública do SUS.

A assistência aos transtornos mentais suscita questões teóricas adicionais ao já complexo tema da provisão de serviços na saúde: maior incerteza e variabilidades nas escolhas de tratamento; grande ambiguidade na definição dos interesses do paciente, consequências sociais e externalidades negativas e custosas. Os transtornos mentais mais graves - esquizofrenia, transtornos bipolares e outras formas de depressão - afetam a cerca de $4 \%$ da população a cada ano e emergem em uma faixa etária relativamente jovem - entre 15 a 30 anos. Os quadros mais graves reduzem a capacidade de consumidor individual e interferem nas suas redes sociais, enfraquecendo as conexões com os que podem atuar como cuidadores ou responsáveis ${ }^{5}$.

Estas características apontam para as grandes dificuldades de mercado de planos privados de assistência à saúde em lidar com o conjunto de problemas associados aos transtornos mentais. 
No sistema de saúde norte-americano, com forte predomínio da indústria de seguro saúde, as empresas privilegiam a seleção de risco, provendo o mínimo de serviços para os pacientes com maior gravidade, entre os quais os pacientes de transtornos mentais para reduzir a seleção adversa ${ }^{5}$.

Em geral, esta situação está também relacionada com o temor do mercado segurador de que a cobertura de serviços de saúde mental possa resultar em altos custos relacionados com psicoterapias intensivas de longo termo e longas internações hospitalares ${ }^{6}$. No caso das psicoterapias, a cobertura tem sido objeto de intensas controvérsias em face da percepção de que sua indicação poderia ser arbitrária e seu uso fortemente afetado pela cobertura através de seguros. Afora a simples recusa de cobrir o tratamento para transtornos mentais, as empresas de seguro de saúde costumam restringir a cobertura a episódios agudos ou impor limites de utilização de procedimentos ou mecanismos de copagamento ${ }^{7}$.

Do ponto de vista da economia da saúde, considera-se que a atenção aos transtornos mentais pode ser particularmente afetada pelo fenômeno denominado risco moral, que ocorre quando as pessoas protegidas de risco se comportam de forma diferente de quando estão totalmente expostas ao risco, gerando altas taxas de utilização ${ }^{5}$. Ou seja: As pessoas, em face de não terem de arcar com o custo total dos procedimentos, se comportam de maneira diversa do que o fariam no caso de pagamento integral.

O segundo fenômeno intrinsecamente, relacionado aos mercados de seguro, que afeta particularmente o cuidado em saúde mental é a seleção adversa, que ocorre quando planos que oferecem a cobertura mais abrangente atraem as pessoas que têm as necessidades de cuidado mais intensas, levando a elevadas taxas de utilização de serviços e, consequentemente, altos custos para os seguradores ${ }^{5}$.

Nos EUA, em face às restrições de cobertura impostas, as pessoas pagam por desembolso direto por uma significativa proporção de serviços e se defrontam eventualmente com perdas financeiras catastróficas ou têm seu cuidado transferido para o setor público quando os gastos excedem seus limites financeiros ${ }^{8}$.

Em contraposição aos Estados Unidos da América, onde o mercado de seguros privados é mais desenvolvido e ocupa um papel mais importante na prestação de serviços de saúde mental, os países europeus apresentam sistemas predominantemente com financiamento público, através de alguma forma de taxação ou contri- buição para previdência pública. No campo dos seguros privados, muitos países permitem explicitamente a exclusão da cobertura de transtornos mentais de natureza crônica ou que levam a intervenções de maior custo 9 .

Como consequência das particularidades do cuidado em saúde mental, sistemas de atenção em saúde mental na maioria dos países ocidentais demandam um amplo papel ao sistema de prestação direta de serviços públicos para a maioria das pessoas com transtornos mentais severos.

$\mathrm{Na}$ Espanha, por exemplo, o papel dos seguros de saúde privados é secundário, mas em expansão, abarcando 13\% da população em 2002. Para 2,7 \% da população, eles representam o principal meio de financiamento da saúde, enquanto $10,3 \%$ os utilizam como um complemento ou suplemento, de sua utilização do sistema público. No que tange à saúde mental, a cobertura é mais limitada com vários planos excluindo psicoterapias e internações psiquiátricas ${ }^{10}$.

No quadro brasileiro, a opção da seleção ativa de risco e a provisão mínima de serviços seriam teoricamente impraticáveis em razão da intervenção da agência reguladora setorial e do quadro institucional mais geral, definido pela Lei 10.216/2001. Entretanto, para responder de modo apropriado ao problema, cabe mostrar o quanto o setor de planos privados de atenção à saúde responde ao desafio da assistência à saúde mental dos segurados.

\section{A Fragmentação do sistema de saúde e o mercado de planos privados de assistência à saúde}

O padrão fragmentado do sistema de saúde brasileiro, assinalado por Londoño e Frenk ${ }^{11}$, tem se consolidado nas últimas décadas. O arranjo fragmentado é composto pelo tripé: gasto privado por desembolso direto, gasto privado em planos de saúde e gasto público pela estrutura federativa do SUS ${ }^{12}$. Em 2009, os gastos privados em saúde eram os mais relevantes, totalizando 54,3 $\%$ dos gastos totais em saúde. Do total de gastos privados, $57,1 \%$ foram por desembolso direto e 41,2 \% com planos privados ${ }^{13}$.

Em março de 2011, existiam 46.634.765 beneficiários de algum plano de saúde de assistência médica, o que correspondia a $24,4 \%$ da população brasileira. A distribuição regional mostra elevada disparidade com a cobertura. Era de $37,8 \%$ no Sudeste, enquanto no Nordeste encontrava-se em 11,1 \%. Em algumas capitais, a cobertura é significativamente alta: 54,1\% em 
Belo Horizonte, 55,6 \% no Rio de Janeiro, 59,8 \% em São Paulo e 75,8 \% em Vitória ${ }^{14}$. Assim, o sistema de saúde brasileiro apresenta a peculiaridade da convivência de um subsistema cuja proposta é o acesso universal e a atenção integral com um grande peso do segmento privado no financiamento e provisão dos serviços.

A aquisição ou acesso aos planos de saúde está fortemente associado à renda. Como mostra a Tabela 1, quanto maior é a renda familiar, maior é a proporção de famílias com planos de saúde ${ }^{15}$.

O padrão estratificado e socialmente desigual do acesso aos planos de saúde torna bastante frágil a tese geral do uso compartilhado da rede de serviços hospitalares, ambulatoriais, diagnóstico e de terapias do sistema público e privado de planos de saúde. A hipótese alternativa desse estudo é que a utilização dos serviços de saúde mental pelos usuários de planos de saúde é residual por duas razões: 1) o dinamismo da oferta de internações psiquiátricas de curta duração e 2) o foco na oferta de cobertura para transtornos mentais agudos pelos planos de saúde.

No setor público, como já assinalado, encontra-se em curso, no campo da assistência pública em saúde mental, um processo de reforma institucional, iniciado nos anos 90, que afeta intensamente a configuração de serviços e dispositivos de cuidado às pessoas acometidas por transtornos mentais graves: a opção pela atenção de base comunitária em detrimento ao procedimento de internação psiquiátrica, que passa a ser, na nova modelagem, uma intervenção de exceção. Concomitante tem sido implantada uma rede de recursos assistenciais de base comunitária. Os prin-

Tabela 1. Percentual de pessoas, na população residente, com cobertura de planos de saúde, segundo as classes de rendimento mensal domiciliar per capita - Brasil - 2008.

\begin{tabular}{lc}
\hline \multicolumn{1}{c}{ Classes de Rendimento } & $\begin{array}{c}\text { \% de Cobertura de } \\
\text { Planos de Saúde }\end{array}$ \\
\hline Sem rendimento e $1 / 4$ do & 2,3 \\
salário mínimo & \\
Mais de $1 / 4$ a $1 / 2$ salário mínimo & 6,4 \\
Mais de $1 / 2$ a 1 salário mínimo & 16,1 \\
Mais de 1 a 2 salários mínimos & 33,7 \\
Mais de 2 a 3 salários mínimos & 54,8 \\
Mais de 3 a 5 salários mínimos & 68,8 \\
Mais de 5 salários mínimos & 82,5 \\
\hline
\end{tabular}

Fonte: IBGE. cipais dispositivos são os Centros de Atenção Psicossocial. A integração da saúde mental com o cuidado primário em saúde tem sido também buscada ${ }^{16,17}$. Esta composição de serviços é considerada, pela maioria dos autores, menos estigmatizante e mais acessível ${ }^{18,19}$.

\section{A regulação da assistência à saúde mental no setor de planos de saúde no Brasil}

Até 1998, a atenção aos transtornos mentais estava excluída da quase totalidade dos contratos das operadoras de planos e seguros privados de assistência à saúde no Brasil. A partir da promulgação da Lei 9.656/98, a cobertura passou a ser obrigatória a todos os contratos celebrados a partir de janeiro de 1999 ou nos planos antigos que foram adaptados em consonância com a $\mathrm{Lei}^{20}$.

A regulamentação da cobertura para os transtornos mentais foi implantada através da Resolução do Conselho de Saúde Suplementar de número 11 (CONSU 11) ${ }^{21}$. Nos planos e seguros do segmento hospitalar foi estabelecido um limite de cobertura de 30 dias de internação por ano de contrato em hospital psiquiátrico ou em hospital geral. No caso do uso prejudicial de álcool e outras drogas, o limite era de 15 dias e as internações só poderiam se dar em hospitais gerais. Ultrapassados estes limites, as operadoras poderiam estabelecer mecanismos de coparticipação crescentes.

A atualização do Rol de Procedimentos e Eventos em Saúde, implantada pela Resolução Normativa $211^{22}$ e pela Instrução Normativa $25^{23}$, que passou a vigorar a partir de Junho de 2010, abandonou a discriminação em dias de cobertura entre internações para usuários de drogas e relativas aos demais transtornos mentais e estabeleceu um limite comum de 30 dias. Foi suprimida também a especificidade de internação em hospitais gerais para as internações motivadas pelo uso prejudicial de álcool e outras drogas. A partir de Janeiro de 2012, o copagamento para internações psiquiátricas que excedam 30 dias por ano de contrato foi limitado em 50\% do valor contratualizado com o prestador ${ }^{24}$.

Ainda no segmento hospitalar, a assistência em hospital-dia, instituída em 1999, teve sua cobertura ampliada em 2010, quando foram suprimidos os limites de duração de tratamento, desde que fossem preenchidos os critérios de diagnóstico estipulados - transtornos mentais e comportamentais devidos ao uso de substância psicoativa, esquizofrenia, transtornos esquizotípicos e transtornos delirantes, transtornos de humor 
(episódio maníaco e transtorno bipolar do humor) e transtornos globais do desenvolvimento.

No segmento ambulatorial, até junho de 2010, além do atendimento médico por psiquiatra, a cobertura mínima estava restrita a 12 sessões de Psicoterapia por ano e seis sessões de terapia ocupacional $^{25}$. Os atendimentos de emergências podiam ser seguidos também de psicoterapias de crise com a duração máxima de 12 semanas e limitadas a 12 sessões por ano de contrato ${ }^{21}$.

A atualização do rol de procedimentos implementada pela ANS em 2010 elevou o número mínimo de consultas por psicólogos e terapeutas ocupacionais para 40 por ano de contrato para um grupo de diagnósticos que incluem as esquizofrenias, os transtornos afetivos bipolares, os transtornos depressivos recorrentes, os transtornos de alimentação e os transtornos globais de desenvolvimento como o autismo. Para outros diagnósticos, como por exemplo, os transtornos neuróticos e os transtornos mentais devidos a substâncias psicoativas, a cobertura ficou restrita a 12 sessões de psicoterapia por psicólogos ou médicos. Os planos de saúde devem ainda cobrir 12 consultas de terapeutas ocupacionais por ano de contrato para alguns diagnósticos específicos, como demências e retardo mental.

Tabela 2. Proporção de empresas sem informação de provisão de serviços para transtornos mentais.

\begin{tabular}{lc}
\hline \multicolumn{1}{c}{ Grupo de Transtornos } & $\begin{array}{c}\text { Ausência de } \\
\text { Cobertura }\end{array}$ \\
\hline $\begin{array}{l}\text { Transtornos mentais leves e } \\
\text { moderados }\end{array}$ & $8,5 \%$ \\
$\begin{array}{l}\text { Neuroses e psicoses graves } \\
\text { Relacionados ao uso de álcool e } \\
\text { outras drogas }\end{array}$ & $17,9 \%$ \\
Demências & $17,9 \%$ \\
\hline
\end{tabular}

Fonte: Requerimento de Informações da ANS 2007-2008

\section{O perfil de oferta e provisão de serviços de saúde mental pelas empresas}

Os resultados da Tabela 2 apontam que, decorridos nove anos desde a obrigatoriedade da atenção assistencial aos eventos decorrentes dos transtornos mentais, continuavam existindo importantes lacunas de disponibilidade de serviços em quase $20 \%$ para os quadros relacionados aos diagnósticos de neuroses e psicoses graves e ao uso de álcool e drogas. Em relação às demências, 22,3 \% das empresas não dispunham de serviços ${ }^{26}$.

A Tabela 3 mostra que a maior disponibilidade de serviços hospitalares é a internação em hospital psiquiátrico $(73,2 \%)$, seguido da internação psiquiátrica em hospital geral $(62,8 \%)$ e do dispositivo hospital-dia $(47,8 \%)$. O porte das operadoras não influencia a oferta de serviços ${ }^{27}$. Os dados de oferta são extremamente homogêneos, com exceção de uma maior oferta de internações em hospitais gerais por parte das operadoras de grande porte.

Apesar das lacunas de oferta observadas nas duas tabelas anteriores, os dados de produção mostravam um crescimento da ordem de $28 \%$ nas internações psiquiátricas do ano de $2005^{28}$ até $2010^{29}$ no sistema de saúde suplementar, segundo o Sistema de Informação de Produtos (SIP), através do qual as operadoras informam sua produção para a ANS. No mesmo período, os beneficiários de planos de saúde com cobertura no segmento hospitalar aumentaram em 61\% (Tabela 4).

Em 2007, foram registradas 10.116 internações psiquiátricas por usuários com planos de saúde realizadas no SUS ${ }^{30}$ em um total de 248.968 internações ${ }^{31}$, o que representa $4,1 \%$ do total. A escala da oferta de internações psiquiátricas no sistema de saúde suplementar parece impactar o nível relativamente baixo de utilização deste procedimento no SUS pelos seus segurados.

Tabela 3. Oferta de serviços de saúde mental por porte das operadoras - segmento hospitalar - em números absolutos e percentagem $\left(\mathrm{N}=749^{*}\right)$.

\begin{tabular}{lrccr}
\hline \multicolumn{1}{c}{ Porte por beneficiários } & Operadoras & Internação em HP & Internação em HG & Hospitais-Dia \\
\hline Pequenas $(<20.000)$ & $488(100 \%)$ & $354(72,5 \%)$ & $303(62,1 \%)$ & $220(45,1 \%)$ \\
Medias $(<100.000)$ & $196(100 \%)$ & $145(74 \%)$ & $120(61,2 \%)$ & $101(51,5 \%)$ \\
Grandes $(>100.000)$ & $65(100 \%)$ & $49(75,4 \%)$ & $47(72,3 \%)$ & $37(56,9 \%)$ \\
Total & $749(100 \%)$ & $548(73,2 \%)$ & $470(62,8 \%)$ & $358(47,8 \%)$ \\
\hline
\end{tabular}

Fonte: Requerimento de Informações da ANS 2007-2008; Anuário ANS 2007. 'Em face de um grupo de operadoras não terem tido suas demonstrações contábeis reportadas no Anuário, a amostra analisada foi de 749 operadoras. 
A comparação da taxa de internações psiquiátricas no setor de saúde suplementar com a taxa do Sistema Único de Saúde em 2010 mostrava uma relevante diferença. Enquanto a taxa na Saúde suplementar foi de 284 por 100 mil usuários, no SUS, a taxa foi de 149 por 100 mil habitantes: $91 \%$ menor (Tabela 5).

A comparação da evolução do número de internações psiquiátricas nos dois sistemas mostra tendências inversas: de redução de $16 \%$ no SUS de 2005 até 2010 e um aumento de $28 \%$ na saúde suplementar no mesmo período (Tabela 6).

Estes resultados parecem indicar uma disparidade entre as políticas de saúde mental nos dois sistemas no que tange a assistência hospitalar. Como se verificou anteriormente, o aumento de internações psiquiátricas não tem acompanhando na mesma proporção o crescimento da base de beneficiários dos planos de saúde com cobertura hospitalar, mas este achado não pode ser relacionado a nenhum procedimento específico de desestímulo da hospitalização.

Convém destacar que tem sido preconizada uma migração da alocação de leitos localizados em hospitais psiquiátricos especializados para enfermarias psiquiátricas em hospitais gerais. Os países com assistência à saúde mental consolidada têm feito a conversão de dispositivos de forma resoluta. A Itália, cujo modelo assistencial é frequentemente tomado como referência pelo Brasil, efetivou a transição completa em relação aos seus leitos públicos ${ }^{32}$. Este movimento organizacional está amparado em fortes evidências que indicam que a atenção centrada em hospitais psiquiátricos, principalmente funcionando desarticulados de uma rede assistencial extrahospitalar, acarreta barreiras ao efetivo cuidado, entre elas o reforço do estigma.

Os dados disponíveis no SIP não apresentam discriminação sobre qual modalidade de dispositivo hospitalar está sendo utilizado para realizar as internações psiquiátricas. As respostas ao requerimento de informações da ANS apontam que é mais comum que o procedimento seja ofertado em hospitais psiquiátricos $(73,2 \%)$ do que em hospital geral $(62,8 \%)$. Com os dados disponíveis não é possível avaliar se a significativa disponibilidade de leitos psiquiátricos em hospital geral referida pelas operadoras está relacionada com a padronização da ANS vigente em 2007-2008 de que as internações dos agravos relacionados à abstinência e a intoxicação pelo uso de álcool e outras drogas deveriam ser realizadas em hospital geral (CONSU 11).

Deste ponto de vista, pode-se considerar como um retrocesso a supressão da restrição contida na CONSU 11, através da RN 211, vigente a partir de junho de 2010. Deste momento em diante, pode-se dizer que o sistema de saúde suplementar não formaliza nenhum mecanismo indutor que possa desestimular o procedimento “internação em hospital psiquiátrico", o que

Tabela 4. Internações psiquiátricas realizadas no setor de saúde suplementar e evolução do número de beneficiários de planos de saúde com cobertura no segmento hospitalar. Brasil 2005-2010.

\begin{tabular}{lccc}
\hline & $\mathbf{2 0 0 5}$ & $\mathbf{2 0 1 0}$ & Variação \% \\
\hline Internações psiquiátricas (1) & 78.720 & 100.650 & $+28 \%$ \\
Beneficiários (2) & 22.024 .778 & 35.490 .641 & $+61 \%$ \\
\hline
\end{tabular}

Fonte: (1) Sistema de Informações de Produtos/ANS. (2) Beneficiários de planos com cobertura para o segmento hospitalar com contratação posterior à Lei 9.656/98 em dezembro de 2005 e dezembro de 2010 (ANS TABNET).

Tabela 5. Comparação da Taxa de Internações psiquiátricas realizadas no SUS e no setor de saúde suplementar - Brasil - 2010 .

\begin{tabular}{lccc}
\hline \multicolumn{1}{c}{ Descritor } & $\begin{array}{c}\text { Internações } \\
\text { Psiquiátricas }\end{array}$ & Beneficiários & $\begin{array}{c}\text { Internações por } \\
\mathbf{1 0 0 . 0 0 0} \text { Beneficiários }\end{array}$ \\
\hline Sistema Único de Saúde (SUS) & $230.629(1)$ & $155.242 .053(3)$ & 149 \\
Saúde Suplementar & $100.650(2)$ & $35.490 .641(4)$ & 284 \\
\hline
\end{tabular}

Fonte: (1) DATASUS (2) Sistema de Informações de Produtos/ANS (3) Diferença entre o total da população brasileira (190.732.694-IBGE) e os beneficiários de planos de saúde (4) Beneficiários de planos com cobertura para o segmento hospitalar com contratação posterior à Lei 9.656/98 em dezembro de 2010 - ANS TABNET. 
marca uma importante diferença com as estratégias implantadas pelo SUS, onde a forte regulação tem ocasionado uma redução importante do número de leitos localizados em hospitais psiquiátricos ${ }^{33}$.

Outro indicador disponível através da consulta feita pala ANS às operadoras é o relativo ao tempo médio de permanência das internações psiquiátricas. Os dados apresentam importante homogeneidade no que tange a limitação dentro do padrão de cobertura integral definido pela ANS de até 30 dias (Tabela 7), sendo que as pequenas e médias operadoras apresentam as médias mais baixas (em torno de dezessete dias). A análise das medianas mostra que metade das operadoras pequenas e médias encontra-se com o tempo médio de internação inferior a 15 dias e as grandes empresas com metade das operadoras com médias abaixo de 20 dias.

O tempo de internação hospitalar tem sido objeto de interesse crescente nos estudos internacionais em face da tendência de que a atenção aos pacientes com transtornos mentais deva ocorrer em "settings" menos restritivos.

Ao longo do processo de desinstitucionalização da assistência psiquiátrica, o número de leitos psiquiátricos e a média de tempo de ocupação dos leitos existentes diminuíram em diversos

Tabela 6. Evolução das internações psiquiátricas no SUS e no setor de saúde suplementar - 2005-2010.

\begin{tabular}{lrcc}
\hline \multicolumn{1}{c}{ Subsistema } & $\mathbf{2 0 0 5}$ & $\mathbf{2 0 1 0}$ & Variação \% \\
\hline Saúde Suplementar & 78.720 & 100.650 & $+28 \%$ \\
SUS & 267.256 & 230.629 & $-16 \%$ \\
\hline
\end{tabular}

Fonte: Sistema de Informações de Produtos/ANS e DATASUS.

Tabela 7. Tempo de permanência (em dias) das internações psiquiátricas por porte das operadoras.

\begin{tabular}{lccc}
\hline \multicolumn{1}{c}{ porte } & operadoras & média & mediana \\
\hline $\begin{array}{l}\text { Pequenas } \\
(<20.000)\end{array}$ & 375 & 16,89 & 15,00 \\
$\begin{array}{l}\text { Medias } \\
(<100.000)\end{array}$ & 152 & 17,58 & 15,00 \\
$\begin{array}{l}\text { Grandes } \\
(>100.000)\end{array}$ & 51 & 26,08 & 20,00 \\
Total & $578^{*}$ & 17,88 & 15,00 \\
\hline
\end{tabular}

Fonte: Requerimento de Informações da ANS 2007-2008. ${ }^{\star} 578$ Operadoras responderam este quesito. países. Uma revisão de seis estudos sobre comparações sobre internações mais curtas ou mais longas mostrou que uma política planejada de internações psiquiátricas mais breves não parece acarretar em problemas de efetividade ${ }^{34}$.

Outra revisão da literatura realizada em 2009 apontou, entretanto, que para que as internações mais curtas funcionem de forma adequada é necessário que o sistema de cuidado extra-hospitalar entre em cena imediatamente após a alta, com respostas qualitativamente adequadas. $\mathrm{O}$ efeito benéfico da curta estada é modulado pelas condições de alta e seguimento ${ }^{35}$.

Um fator importante de análise sobre a possível efetividade da opção regulatória da ANS no que tange aos usuários com transtornos severos é a integração das unidades de internação com os demais dispositivos externos, para garantir a continuidade de cuidados. Entretanto, como se pode observar na Tabela 8 , as operadoras não têm enfatizado programas para usuários que sofreram internações psiquiátricas.

O processo de desospitalização da assistência psiquiátrica envolve diversas variáveis, sendo uma delas o fator econômico. Mesmo em países que reduziram significativamente o papel da internação psiquiátrica, os custos hospitalares seguem sendo a principal fonte de dispêndio através de gastos diretos com os transtornos mentais severos ${ }^{36}$.

A estratégia desenvolvida pela ANS para as internações envolve dois constrangimentos. O primeiro responde à necessidade de controle financeiro por parte das operadoras, que impelidas a efetivar a cobertura aos transtornos mentais, mantém um determinado controle sobre o risco. O segundo funciona como fator limitador

Tabela 8. Oferta de programas específicos para egressos de internações psiquiátricas por porte das operadoras - em números absolutos e percentagem.

\begin{tabular}{lccc}
\hline \multicolumn{1}{c}{ porte } & Operadoras & \multicolumn{1}{c}{ Sim } & Não \\
\hline $\begin{array}{l}\text { Pequenas } \\
(<20.000)\end{array}$ & $488(100 \%)$ & $20(4,1 \%)$ & $468(95,9 \%)$ \\
$\begin{array}{l}\text { Medias } \\
(<100.000)\end{array}$ & $196(100 \%)$ & $13(6,6 \%)$ & $183(93,4 \%)$ \\
$\begin{array}{l}\text { Grandes } \\
(>100.000)\end{array}$ & $65(100 \%)$ & $04(6,2 \%)$ & $61(93,8 \%)$ \\
Total & $749(100 \%)$ & $37(5,0 \%)$ & $712(95 \%)$ \\
\hline
\end{tabular}

Fonte: Requerimento de Informações da ANS 2007-2008. Anuário ANS 2007. 
sobre a longa permanência hospitalar, que para resultar em benefício para os usuários é necessário analisar se a limitação não está ocasionando altas prematuras motivadas pela limitação de cobertura. Para fins de desembolso direto das famílias, o período que exceder 30 dias pode resultar em significativo ônus financeiro para uma parcela dos usuários de planos de saúde, podendo chegar a apresentar as características de gasto catastrófico, como se convencionou chamar os gastos que excedem intensamente a capacidade financeira das famílias, ou acarretar a procura do serviço público para continuação do tratamento.

Considere-se que o período de 30 dias está dentro de padrões aceitáveis para a evolução de uma crise em ambiente hospitalar até a data da alta, apesar de muitos países operarem com um tempo médio de internação superior ${ }^{37}$. Entretanto, por exemplo, na Itália, estudo de âmbito nacional, realizado em 2001, apontou que a percentagem de pacientes em "revolving door", definidos como os pacientes que tiveram três ou mais admissões na mesma unidade psiquiátrica pública de internação no período de um ano, foi de $8,7 \%$ do total de internados. Além disso, $22,3 \%$ dos pacientes internados estavam em sua segunda internação no mesmo ano ${ }^{38}$. Como a cobertura da ANS se restringe a 30 dias por ano de contrato, a soma dos dias de mais de uma internação pode impedir a cobertura integral por parte da operadora.

No período analisado, que vai de meados de 2007 até começo de 2008, o dispositivo hospitaldia ainda se encontrava em processo de expansão. Na Resolução Normativa 211/2010 é definido como "recurso intermediário entre a internação e o ambulatório, que deve desenvolver programas de atenção e cuidados intensivos por equipe multiprofissional, visando substituir a internação convencional..." (Parágrafo $1^{\circ}$ do artigo 18).

Pode-se depreender pela definição, adotada da Portaria SNAS 224 de 1992 do Ministério da Saú$\mathrm{de}^{39}$, e pelo seu posicionamento no segmento hospitalar dos planos de saúde, que o dispositivo está direcionado para pacientes em situação aguda ou de crise, funcionando o dispositivo hospital-dia como uma alternativa à internação convencional, mas não direcionado para portadores de transtornos mentais severos em quadro persistente, que necessitam de abordagem específica, voltada para a reabilitação psicossocial. A literatura disponível sugere que a utilização de hospitais-dia para pacientes em crise pode ser tão efetiva quanto a internação usual, com vantagens no que tange à capacidade de reintegração social e à autono- mia por ocasião da alta ${ }^{40}$. Entretanto, no panorama internacional, esta modalidade de cuidado vem se tornando superada por novas abordagens, como a atenção domiciliar ${ }^{41}$ e vem assumindo um papel residual no Sistema Único de Saúde brasileiro, no qual a atenção diária para pacientes com transtornos severos, tanto para situações de crise, quanto em situação de atenção de longo curso, vem sendo desenvolvida no âmbito dos Centros de Atenção Psicossocial.

Esta disposição está explicitada de forma categórica em documento do Ministério da Saúde de 2007, conforme trecho abaixo:

"O hospital-dia de saúde mental [...] é hoje em dia um serviço em processo de superação, especialmente face ao papel desempenhado pelos Centros de Atenção Psicossocial [...], o Ministério da Saúde deverá recadastrar nos próximos anos os hospitais-dia que já funcionam como CAPS, e rediscutir aqueles que permanecerem com um perfil hospitalar, os quais têm mostrado baixa efetividade na reintegração social dos pacientes egressos de internações. Espera-se que a implantação de serviços comunitários (CAPS e ambulatórios) e a expansão da atenção psiquiátrica em hospitais gerais conduza à superação progressiva deste dispositivo, que teve importância histórica na mudança do modelo de atenção em saúde mental".

A intenção dos formuladores da política do SUS, ao diminuir a ênfase na implantação e desenvolvimento dos hospitais-dia, parece estar relacionada com a ligação implícita dessas estruturas com os hospitais psiquiátricos e pelas dificuldades de se constituírem como referência para o restante da rede assistencial em um modelo que se pretende marcado pela territorialização e pela integração com a atenção primária.

Porque a ANS, em sua revisão do rol que foi implantada em junho de 2010, manteve a modelagem dos hospitais dia como única modalidade de cuidado extra-hospitalar, além das consultas de cunho ambulatorial, apesar do flagrante descolamento com as políticas desenvolvidas pelo SUS? Provavelmente porque acarretaria na necessidade da constituição pelas operadoras de novos serviços, de modalidade diversa dos já constituídos, com o consequente aumento das despesas operacionais, mas também e fundamentalmente porque implicaria na assunção da cobertura mais abrangente voltada para pacientes com transtornos severos persistentes, no espectro da cronicidade e do cuidado de longo curso.

Um dos mais importantes achados na epidemiologia da esquizofrenia em termos de impli- 
cações para os serviços é que uma alta proporção de pacientes pode sofrer de incapacidades sociais moderadas e severas. As implicações para o planejamento da atenção em saúde mental é a necessidade de provisão de intervenções integradas de longo prazo ${ }^{42}$. O padrão de utilização de serviços costuma ser heterogêneo e influenciado pela gravidade dos $\operatorname{casos}^{43}$.

Os programas que devem ser oferecidos para esta população desejavelmente vão além de consultas ambulatoriais médicas ou de outras categorias profissionais. Os tratamentos efetivos prevêem o controle e/ou redução dos sintomas através da psicofarmacologia, que, entretanto, não é suficiente para um segmento importante dos pacientes, onde a combinação com psicoterapias e intervenções psicossociais resulta em aumento de eficácia ${ }^{18,44}$.

Assim, a carteira de serviços oferecida pelas empresas de planos de saúde reguladas pela ANS pode estar limitada em eficácia para um segmento de usuários com um determinado perfil. O provável efeito das medidas restritivas é a absorção dos gastos excedentes pelas famílias ou a transferência do cuidado para o SUS dos pacientes cujas necessidades de serviços extrapolem a modelagem prevista. Neste perfil de pacientes podem se encontrar os casos de transtornos mais severos e os de longo curso.

\section{Conclusões}

A ANS tem ampliado a regulação sobre as operadoras dos planos de saúde no que tange à assistência dos transtornos mentais desde a sua criação em 2000. Ainda assim, este artigo identifica falhas na oferta de serviços para pacientes com quadros de demências, neuroses, psicoses graves e ao uso prejudicial de álcool e drogas em fins da década passada em empresas pequenas, grandes e médias. Chama atenção também a oferta de internações psiquiátricas em expansão. A comparação com o SUS indica uma taxa de internação mais elevada no setor de saúde suplementar. Qual o significado normativo dessa alta prevalência das internações psiquiátricas no mercado de planos privados de assistência à saúde?

Pode-se dizer que a população detentora de planos de saúde está exposta ao risco decorrente da internação em hospital psiquiátrico. Por não informar as questões inerentes a este risco, a ANS não oferece as condições de uma escolha suficientemente informada para os usuários selecionarem os locais mais adequados para o cuidado institucional das crises derivadas dos transtornos mentais.

Outro possível efeito indesejado é a indução de padrões de consumo de serviços de saúde mental, o que pode gerar expectativas na população em geral, levando ao recrudescimento de crenças difusas sobre os valores terapêuticos do isolamento e da hospitalização.

Outro aspecto preocupante e contraditório é que a regulação da assistência à saúde mental do mercado de seguro saúde brasileiro apresenta similitudes ao observado em outros países ao não inibir o uso do mecanismo de copagamento específico para saúde mental, relacionado com as internações psiquiátricas. Apresenta também uma limitação de quantitativo de consultas ambulatoriais não médicas, que é mais intensa em alguns grupos diagnósticos. Nesta situação, encontra-se um segmento altamente vulnerável, os usuários que fazem uso prejudicial de álcool e de outras drogas, que só podem se beneficiar de 12 consultas psicoterápicas por ano de cobertura. Destaque-se a significativa ampliação de cobertura de consultas de psicologia e terapia ocupacional (de 12 para 40 por ano de contrato) para outros diagnósticos de transtornos severos como o autismo e os transtornos do espectro da esquizofrenia.

Em que medida a normatização instituída pela ANS está desenhada para reduzir mecanismos de mercado que afetam a provisão da assistência à saúde mental pelas empresas de planos e seguros de saúde, ou se está favorecendo barreiras de acesso e lacuna assistencial, é uma questão em aberto carecendo de estudos posteriores.

Uma possível resposta está associada às limitações deste estudo. O Sistema de Informações de Produtos da ANS não oferece informações sobre a natureza e a estrutura dos serviços disponibilizados pelas operadoras, permitindo apenas analisar o quantitativo de produção. Foram estudadas ainda as informações da consulta da ANS junto às operadoras de planos de saúde, respondida no período de agosto de 2007 a março de 2008, que não refletem o possível impacto das modificações da regulação ocorridas deste momento em diante. 


\section{Colaboradores}

PF Silva e NR Costa participaram igualmente de todas as etapas do artigo.

\section{Agradecimento}

Agradecemos a Agência Nacional de Saúde Suplementar pela disponibilização dos dados referentes ao Sistema de Informações de Produtos do ano de 2010 e ao Requerimento de Informações sobre a atenção em saúde mental de 2007.

\section{Referências}

1. Costa NR. O Regime Regulatório e o Mercado de Planos de Saúde no Brasil. Cien Saude Colet 2008; 13(5):1453-1462.

2. Bahia L. Planos privados de saúde: luzes e sombras no debate setorial dos anos 90. Cien Saude Colet 2001; 6(2):329-340.

3. Ocké-Reis CO. O desafio da ANS frente à concentração dos planos de saúde. Cien Saude Colet 2007; 12(4):1041-1050.

4. Costa NR, Castro AJW. O Regime Regulatório e Estrutura do Mercado de Planos de Assistência à Saúde no Brasil. Regulação e Saúde. Rio de Janeiro: Agência Nacional de Saúde Suplementar; 2003. Volume 3:49-64.

5. Frank RG, McGuire TG. Economics and Mental Health. In: Handbook of Health Economics. Amsterdan: Culyer A.J. \& Newhouse J.P. Elsevier; 2000. v. 1 B.

6. Barry CL, Frank RG, McGuire TG. The costs of mental health parity: still an impediment? Health Affairs 2006; 25(3):623-634.

7. U.S. Department of health and human services. Mental Health: A Report of the Surgeon General. Rockville, MD: U.S. Department of Health and Human Services, Substance Abuse and Mental Health Services Administration, Center for Mental Health Services, National Institutes of Health, National Institute of Mental Health; 1999.

8. Zukevas SH, Selden TM. Mental Health and Family Out-of-Pocket Expenditure Burdens. Medical Care Research and Review 2010; 67(2):194-212.

9. Knapp M, McDaid D. Financing and Funding Mental Health Care Services. In: Knapp M, McDaid D, Mossialos E, Thornicroft G, organizadores. Mental Health Policy and Practice across Europe. European Observatory on Health Systems and Policies Series. New York: The McGraw-Hill Companies; 2007.

10. Salvador-Carulla L, Garrido M, McDaid D, Haro JM. Financing Mental Health in Spain: Context and Critical Issues. Eur J Psychiat 2006; 20(1):29-44.

11. Londoño JL, Frenk J. Structured Pluralism: Towards an Innovative Model for Health System Reform. Latin America Health Policy 1997; 41(1):1-36.

12. Costa NR. México: Estudo de Caso. In: Regulação do Setor Saúde nas Américas: As Relações entre o Público e o Privado numa Abordagem Sistêmica. Brasília: Organização Pan-Americana de Saúde (OPAS); 2006. p. 375-392.

13. World Health Organization (WHO). Global Health Observatory Data Repository. [acessado 2011 jul 20]. Disponível em: www.who.int/research/en/

14. Brasil. Agencia Nacional de Saúde Suplementar. Caderno de Informação da Saúde Suplementar. Junho de 2011.

15. Instituto Brasileiro de Geografia e Estatística (IBGE). Um Panorama de Saúde no Brasil: Acesso e Utilização dos Serviços, Condições de Saúde e Fatores de Risco e Proteção à Saúde. Rio de Janeiro: Instituto Brasileiro de Geografia e Estatística (IBGE); 2008.

16. Brasil. Ministério da Saúde (MS). Saúde Mental no SUS: Acesso ao Tratamento e Mudança do Modelo de Atenção - Relatório de Gestão 2003-2006. Coordenação Geral de Saúde Mental. Brasília: Ministério da Saúde (MS); 2007. 
17. Brasil. Ministério da Saúde (MS). Saúde Mental no SUS: As Novas Fronteiras da Reforma Psiquiátrica. Relatório de Gestão 2007-2010. Coordenação Geral de Saúde Mental, Álcool e Outras Drogas. Brasília: Ministério da Saúde (MS); 2011.

18. Organização Mundial da Saúde (OMS). Saúde Mental: Nova Concepção, Nova Esperança. Genève: Organização Mundial da Saúde (OMS); 2001.

19. World Health Organization (WHO). Joint report: Integrating mental health into primary care - a global perspective. Geneva: World Health Organization publication; 2008.

20. Brasil. Lei no 9.656, de 3 de junho de 1998. Dispõe sobre os planos e seguros privados de assistência à saúde. Diário Oficial da União 1998; 3 jun.

21. Brasil. Conselho de Saúde Suplementar. Resolução CONSU número 11. Dispõe sobre a cobertura aos tratamentos dos transtornos psiquiátricos. Diário Oficial da União 1998; 4 nov.

22. Brasil. Agência Nacional de Saúde Suplementar (ANS). Resolução normativa No 211. Atualiza o Rol de Procedimentos e Eventos em Saúde, que constitui a referência básica para cobertura assistencial mínima nos planos privados de assistência à saúde. Diário Oficial da União 2010; 11 jan.

23. Brasil. Agência Nacional de Saúde Suplementar (ANS). Instrução Normativa número 25. Dispõe sobre as Diretrizes de Utilização e as Diretrizes Clínicas de alguns procedimentos listados no Anexo da Resolução Normativa no 211. Diário Oficial da União 2010; 12 jan.

24. Brasil. Agência Nacional de Saúde Suplementar (ANS). Resolução Normativa - RN no 262, de 1 de agosto de 2011. Atualiza o Rol de Procedimentos e Eventos em Saúde previstos na RN no 211, de 11 de janeiro de 2010. Diário Oficial da União 2011; 1 ago.

25. Brasil. Agência Nacional de Saúde Suplementar (ANS). Rol de Procedimentos e Eventos em Saúde: Resolução Normativa 167/08. Rio de Janeiro: Agência Nacional de Saúde Suplementar (ANS); 2008.

26. Brasil. Agência Nacional de Saúde Suplementar (ANS). Banco de Dados do Requerimento de Informações sobre a atenção em saúde mental, 2007-2008. [Disponibilizado pela Agência Nacional de Saúde Suplementar (ANS)].

27. Brasil. Agência Nacional de Saúde Suplementar (ANS). Anuário ANS - Aspectos Econômico-financeiros das operadoras de Planos de Saúde. Ano Base 2007.

28. Brasil. Agência Nacional de Saúde Suplementar (ANS). Nota Técnica 005/2008/GGTAP/DIPRO/ANS sobre a assistência prestada aos portadores de transtornos mentais na saúde suplementar. Rio de Janeiro: Agência Nacional de Saúde Suplementar (ANS); 2008.

29. Brasil. Agência Nacional de Saúde Suplementar (ANS). Consolidado do Sistema de Informações de Produtos 2010. [Disponibilizado pela Agência Nacional de Saúde Suplementar (ANS)].

30. Brasil. Agência Nacional de Saúde Suplementar (ANS). ANS TABNET. [acessado 2011 jul 20]. Disponível em: http://www.ans.gov.br/anstabnet/anstabnet/materia_novo.htm.
31. Brasil. Ministério da Saúde (MS). Departamento de Informática do SUS (DATA-SUS). Dados oriundos do Sistema de Informações Hospitalares do SUS (SIH/SUS). [acessado 2011 jul 20]. Disponível em: http://tabnet.datasus.gov.br/cgi/deftohtm.exe?sih/ cnv/sxuf.def

32. Girolamo G, Bassi M, Neri G, Ruggeri M, Santore G, Picardi A. The Current State of Mental Health Care in Italy: Problems, Perspectives, and Lessons to Learn. Eur Arch Psychiatry Clin Neurosci 2007; 257(2):83-91.

33. Brasil. Ministério da Saúde (MS). Saúde Mental em Dados 8. Secretaria de Atenção à Saúde. Departamento de Ações Programáticas Estratégicas. Coordenação Geral de Saúde Mental, Álcool e Outras Drogas. Ano VI, no 8, janeiro de 2011.

34. Alwan NA, Johnstone P, Zolese G. Lenght of Hospitalisation for People with Severe Mental Illness. Cochrane Database Syst Rev. 2008; 23 (1):CD000384.

35. Capdevielle D, Boulenger JP, Villebrun D, Ritchie K. Durées d' hospitalisation des Patients souffrant de Schizophrénie: Implication des Systèms de Soin et Conséquences Médicoéconomiques. Encephale 2009; 35(4):394-399.

36. Knapp M, Chisholm D, Astin J, Lelliott P, Audini B. The cost consequences of changing the hospitalcommunity balance: the mental health residential care study. Psychol Med 1997; 27(3):681-692.

37. World Health Organization (WHO). Regional Office for Europe. Policies and practices for mental health in Europe - meeting the challenges. Copenhagen: World Health Organization (WHO); 2008.

38. Girolamo G, Barbato A, Bracco R, Gaddini A, Miglio R, Morosini P, Norcio B, Picardi A, Rossi E, Rucci P, Santone G, Dell-Acqua G. Characteristics and activities of acute psychiatric in-patient facilities: national survey in Italy. Br J Psychiatri 2007; 191:170-177.

39. Brasil. Ministério da Saúde (MS). Portaria SNAS/ MS 224 de 29 de janeiro de 1992. Dispõe sobre a organização dos serviços de saúde mental. Diário Oficial da União 1992; 29 jan.

40. Kallert TW, Pribe S, McCabe R, Kiejna A, Rymaszewska J, Nawka P, Ocvar L, Raboch J, Stárková-Kalisová L, Koch R, Schutzwohl M. Are Day Hospitals Effective for Acutely Ill Psychiatric Patients? A European Multicenter Randomized Controlled Trial. J Clin Psychiatry 2007; 68(2):278-287.

41. Gutierrez-Recacha P, Chisholm D, Haro JM, Salvador-Carulla L, Ayuso-Mateos JL. Cost-effectiveness of different clinical interventions for reducing the burden of schizophrenia in Spain. Acta Psychiatrica Scandinavica 2006; Supl.(432):29-38.

42. European Observatory on Health Systems and Policies. Mental Health Policies in Europe Euro Observer. Autumn 2009; 11(3).

43. Thornicroft G, Tansella M. The implications of epidemiology for service planning in schizophrenia. In: Murray RM, Jones PB, Susser E, OS JV, Cannon M, edited. The Epidemiology of Schizophrenia. São Paulo: Cambridge University Press; 2003. 
44. Pezzimenti M, Haro JM, Ochoa S, González JL, Almenara J, Alonso J, Moreno B, Muñoz PE, Jáuregui VM, Salvador-Carulla L. Assessment of Service Use Patterns in Out-patients with Schizophrenia: a Spanish Study. Acta Psychiatr Scand 2006:114 (Suppl. 432):12-18.

45. Gutierrez-Recacha P, Chisholm D, Haro JM, Salvador-Carulla L, Ayuso-Mateos, JL. Cost-Effectiveness of Different Clinical Interventions for Reducing the Burden of Schizophrenia in Spain. Acta Psychiatr Scand 2006; 114 (Suppl. 432):29-38.

Apresentado em 08/09/2011

Aprovado em 30/09/2011

Versão final apresentada em 05/10/2011 